\title{
Impact of Clinical Pharmacy Specialists on the Design and Implementation of a Quality Improvement Initiative to Decrease Inappropriate Medications in a Veterans Affairs Emergency Department
}

\author{
Jason M. Moss, PharmD, CGP; William E. Bryan, III, PharmD, BCPS; Loren M. Wilkerson, MD; \\ George L. Jackson, PhD, MHA; Ryan K. Owenby, PharmD; Courtney Van Houtven, PhD; \\ Melissa B. Stevens, MD; James S. Powers, MD; Camille P. Vaughan, MD, MS; \\ William W. Hung, MD, MPH; Ula Hwang, MD, MPH; Alayne D. Markland, DO, MSc; \\ Gerald McGwin, MS, PhD; and Susan Nicole Hastings, MD, MHS
}

\begin{abstract}
BACKGROUND: As the proportion of older adult patients who interface with the health care system grows, clinical pharmacy specialists (CPS) have a pivotal role in reducing potentially inappropriate medication (PIM) use in this population.
\end{abstract}

OBJECTIVES: To (a) describe CPS involvement in the design and implementation of a quality improvement (QI) initiative to decrease PIM prescribing in a Veterans Affairs (VA) emergency department (ED) and (b) report on changes in PIM prescribing before and after the initiative.

METHODS: Enhancing Quality of Prescribing Practices for Veterans Discharged from the Emergency Department (EQUiPPED) is an ongoing multisite QI project that aims to decrease ED PIM prescribing. We used a mixed-method approach that applied qualitative and quantitative measures in describing the CPS role and evaluating PIM rates. PIMs were defined using the 2012 Beers Criteria. We reported monthly PIM rates in patients aged 65 years and older who were discharged from the ED from January 2012 to November 2014. A piecewise, nonlinear regression model evaluated the pattern in PIM prescriptions over time.

RESULTS: At the Durham, North Carolina, VA Medical Center, a total of 4 CPS were involved with tailoring the design and implementation of the EQUiPPED intervention for local use. CPS input led to 3 key innovations: academic detailing performed by a physician-CPS pair, medication alert messages identifying medications as PIMs in the computerized patient record system, and automated reports describing the frequency and type of PIMs prescribed by each ED provider. Between February 2013 and November 2014, 73 ED providers received the academic detailing. The ED facility experienced a relative reduction of $47.5 \%$ in the rate of PIM prescribing over the observation period.

CONCLUSIONS: This QI project resulted in a meaningful decrease in PIM prescribing in older ED adults. CPS contributions to QI can extend beyond pharmacotherapy and provider education to also include information technology tools using formulary management expertise.

J Manag Care Spec Pharm. 2016;22(1):74-80

Copyright $\odot 2016$, Academy of Managed Care Pharmacy. All rights reserved.

\section{What is already known about this subject}

Optimizing medication use to treat conditions while avoiding adverse drug events is often challenging, particularly in the older adult who is already at an increased risk of harm from pharmacologic side effects.

Adults aged 65 years and older are at increased risk of drugrelated problems because of suboptimal prescribing in the emergency department (ED).

Several studies have shown benefit from involvement of clinical pharmacy specialists (CPS) in the ED, as well as a variety of other clinical areas

\section{What this study adds}

The impactful role of CPS as a part of an ED-based quality improvement (QI) program resulted in a meaningful decrease in potentially inappropriate medication (PIM) prescribing in older adults and may serve as a model for other ED facilities to decrease PIM prescribing.

There was a relative reduction of $47.5 \%$ in the rate of PIM prescribing in older adults discharged from a Veterans Affairs ED. The monthly rate of PIM prescriptions varied but revealed a decreasing trend up to an inflection point where the rate declined more sharply to the lowest rates of PIM prescribing at that point in the project.

T he term "silver tsunami" describes the dramatic increase in the population size of older adults interfacing with health care systems. ${ }^{1}$ Older adults often receive medication therapy as a cornerstone of care. Optimizing medication use to treat conditions while avoiding adverse drug events is often challenging, particularly in the older adult who is already at an increased risk of harm from pharmacologic side effects.

The challenges related to safe medication therapy in older adults are particularly apparent in emergency departments 
(EDs). Older adults who are prescribed medications in the ED are at increased risk of drug-related problems because of suboptimal prescribing in this population., 2,3 In a Veteran Affairs (VA) retrospective cohort study, approximately $31 \%$ of older adults discharged from the ED were prescribed a suboptimal medication; of these discharged adults, $42 \%$ experienced ED re-presentation, hospitalization, or death within the subsequent 90 days. ${ }^{2}$ One way to measure potentially inappropriate medications (PIMs) is via the Beers Criteria, which were updated by the American Geriatrics Society in 2012 to include evidence-based recommendations for identifying medications that are high risk in older adults. ${ }^{4}$ The Beers Criteria has been used extensively in PIM research in various settings including the ED. ${ }^{5-7}$

Several studies have shown benefit from the involvement of clinical pharmacy specialists (CPS) in the ED, as well as in a variety of other clinical areas..$^{8-12}$ Few studies, however, have described innovative roles for pharmacists within the context of a multidisciplinary quality improvement (QI) initiative. The primary purpose of this clinical report is to describe CPS involvement in the design and implementation of a QI intervention to improve prescribing practices in the ED at $1 \mathrm{VA}$ facility. The secondary aim is to report results of the change in PIM prescribing before and after project initiation.

\section{Methods}

\section{Overview}

Enhancing Quality of Prescribing Practice for Veterans Discharged from the Emergency Department (EQUiPPED) is an ongoing, multisite QI initiative that aims to decrease ED PIM prescribing. The primary goal of the project is to reduce ED prescribing of PIMs for veterans aged 65 years and older at the time of ED discharge. ${ }^{13}$ The EQUiPPED intervention is based on 3 main components: provider education, clinical decision support, and provider feedback on prescribing practices. The intervention was designed to be flexible, with each site tailoring the individual project components to meet the needs of its specific VA ED. Following VA regulations, the EQUiPPED initiative was deemed QI and therefore did not require approval from an institutional review board.

\section{Setting}

The Veterans Health Administration is the largest integrated health care system in the United States, providing care to more than 8 million veterans each year. The Durham, North Carolina, VA Medical Center (VAMC), one of 150 VA medical centers, is a tertiary care and research facility that has an academic affiliation with Duke University. The Durham VAMC ED provides care for approximately 32,000 patient visits annually; of these, approximately $40 \%$ are aged 65 years and older. ${ }^{14}$ ED providers who prescribe medications include attending physicians, advanced practice providers, internal medicine residents, and emergency medicine residents. All ED discharge medications are ordered through the Computerized Patient Record System (CPRS), which serves as the VA electronic health record system.

\section{Qualitative Measure to Determine CPS Role}

The Durham VAMC has approximately 30 CPS in a variety of clinical settings, including formulary management, ambulatory care, geriatrics, internal medicine, infectious disease, nutrition support/critical care, drug information, oncology, and anticoagulation. VA CPS are advanced practice, nonphysician providers with expertise in medication management with prescriptive authority. Qualitative methods used to described the CPS role in EQUiPPED at the Durham VAMC included individual, semistructured interviews with the CPS involved in the project and the site principal investigator. Interdisciplinary project team members were then consulted as a group to provide additional insights into CPS roles. Interview data were collated by a research coordinator. The goals were to identify the specific ways the intervention was tailored for local use in Durham and the role that CPS played in designing and/or implementing these changes.

\section{PIM Measures}

A medication was classified as an "ED discharge medication" if the medication order specified the ED as the ordering clinic location and the prescriber was an ED provider. We included all ED discharge medications prescribed to patients aged 65 years and older discharged from the Durham VAMC during the observation period. PIMs were defined by the American Geriatrics Society 2012 Beers Criteria Update as category 1 medications adapted from table 2 in the update to avoid in all older adults. ${ }^{4}$ Nonsteroidal anti-inflammatory drugs (NSAIDs) are also defined as PIMs by the Beers Criteria with a specific recommendation to avoid chronic use. In order to standardize the definition of chronic use in our QI project, we included NSAIDs as PIMs ordered with a days supply of 30 or greater. ${ }^{13}$

\section{Data Collection and Analysis}

Qualitative data from the individual interviews were reviewed as they became available. Weekly QI team meetings lasting approximately 1 hour were used to review the interview data and to identify areas that needed attention with regards to the overall progression of the QI project. Quantitative data were measured using monthly PIM rates (dependent variable) defined as new PIMs over total discharge medications prescribed to patients aged 65 and older. The EQUiPPED project team set a benchmark goal of reducing PIMs to $5 \%$ or less based on previous research and baseline PIM rates. ${ }^{13}$ To evaluate patterns in monthly PIM rates over time, a piecewise, nonlinear regression model was selected, which allowed for multiple linear relationships to be fit to the independent variable (time) and the dependent variable (monthly rate of PIMS) to elucidate an inflection 


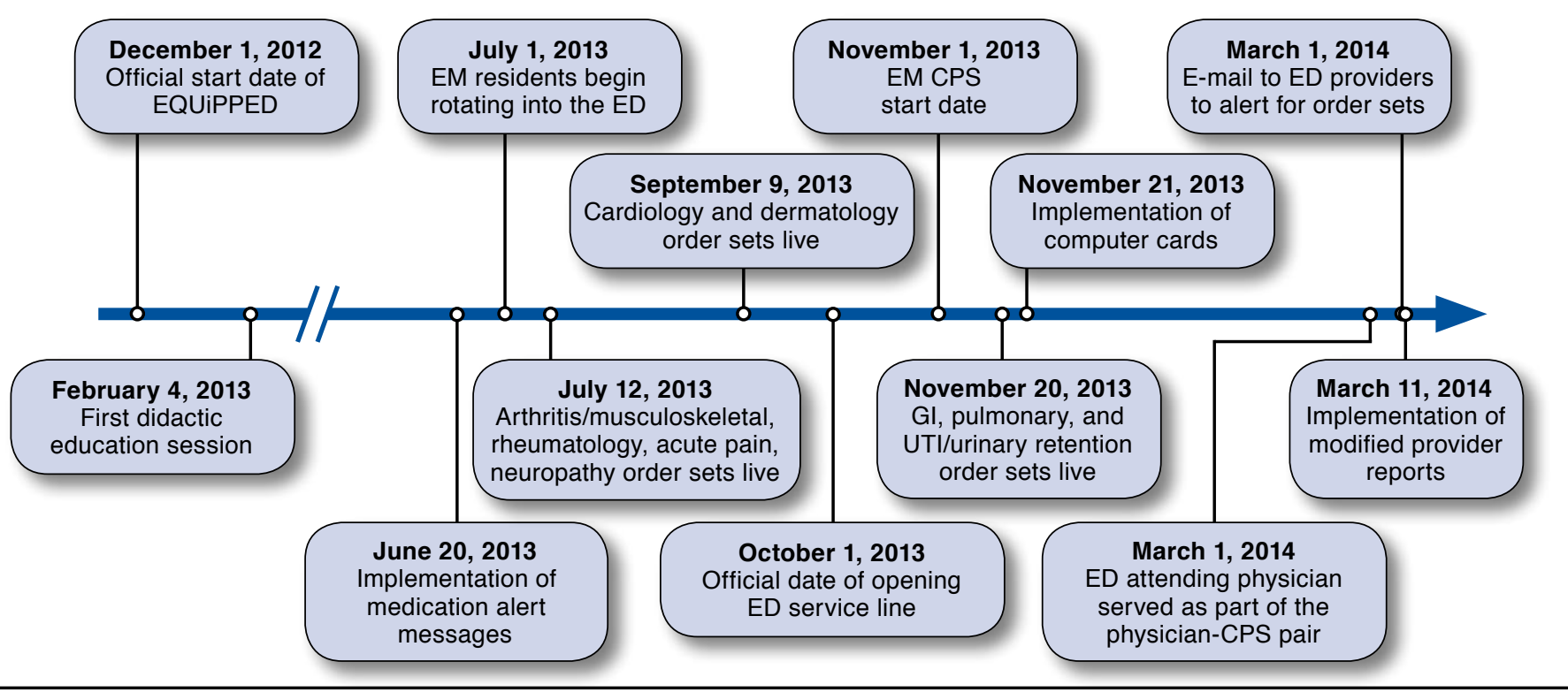

$C P S=$ clinical pharmacy specialist ED = emergency department; EM = emergency medicine; EQUiPPED = Enhancing Quality of Prescribing Practices for Veterans Discharged from the Emergency Department; GI = gastrointestinal; UTI = uninary tract infection.

point in the data. January 2012 to January 2013 was used for baseline purposes to obtain PIM rates before the EQUiPPED interventions. The QI period was defined as February 2013, which coincided with the first ED providers trained through November 2014 (at the writing of the manuscript for this article, the project was still ongoing). Analyses were conducted with SAS version 9.2 (SAS Institute, Cary, NC).

\section{Results}

\section{Key CPS Innovations}

At the Durham VAMC, 4 CPS were involved with tailoring the design and implementation of the intervention for local use. The CPS included a geriatric faculty clinician, a geriatric clinical pharmacist, a formulary management CPS with training in geriatrics, and an emergency medicine (EM) CPS. Project leaders and team members reported that knowledge of local data systems, EM, geriatric pharmacotherapy, and relationships built with ED providers were key in implementation. CPS input led to 3 key innovations: academic detailing performed by a physician-CPS pair, automated reports describing the frequency and type of PIMs prescribed by each ED provider, and medication alert messages identifying medications as PIMs at computerized order entry. The key innovations were developed as the QI initiative progressed and as such were implemented at various time points (Figure 1).

Physician-CPS Academic Detailing. Between February 2013 and November 2014, 73 ED providers received academic detailing by the physician-CPS pair. Of the 73 providers, 10 were physician attendings, 60 were medical residents, and 3 were advanced practice providers. The core attending ED providers were prioritized to receive individual detailing sessions early in the QI project including newly hired providers. Internal medicine residents received the session at the beginning of their 1-month ED rotations. Each provider completed a short questionnaire before starting the session that measured their current prescribing habits, knowledge of the Beers Criteria, and perceived barriers to appropriate prescribing for older adults. The educational academic detailing highlighted age-related changes in the older adult and appropriate prescribing. Specifically, core concepts included pharmacokinetics and pharmacodynamics changes with aging, a review of the 2012 AGS Beers Criteria highlighting PIMs, and safer alternatives for commonly prescribed PIMs. Additionally, detailing focused on commonly prescribed PIMs at our local VA ED that had been elucidated from previous research. ${ }^{2}$ In addition to education, each detailing session was tailored to include case discussions or review of specific PIMs based on the discussions among the providers and the physician-CPS pair. Each provider received a laminated pocket card of the 2012 Beers Criteria along with an iTunes gift card to purchase the educational mobile app iGeriatrics. Lastly, education cards were placed on workstation computers in order to bring to the providers attention commonly prescribed PIMs. 


\section{Impact of Clinical Pharmacy Specialists on the Design and Implementation of a Quality Improvement Initiative to Decrease Inappropriate Medications in a Veterans Affairs Emergency Department}

\section{FIGURE 2 Revised Provider Feedback Form}

EQUiPPED Provider Feedback Form

Provider Name: Provider 2

Goal: To decrease the percentage of potentially inappropriate medications (PIMs) prescribed to veterans 65 years and older at the time of discharge from

the emergency department (ED) to 5\%.

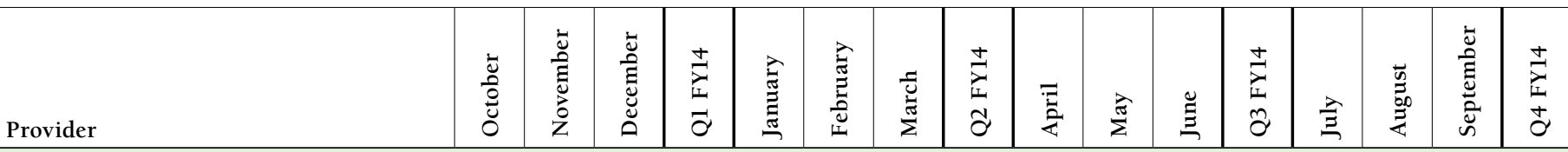

\section{Provider 1}

Number of patients $\geq 65$ years seen in ED

Total number of discharge Rx for patients $\geq 65$

Number of Beers list Rx prescribed

Percentage of Beers list Rx prescribed

Provider 2

Number of patients $\geq 65$ years seen in ED

Total number of discharge Rx for patients $\geq 65$

Number of Beers list Rx prescribed

Percentage of Beers list Rx prescribed

\section{Provider 3}

Number of patients $\geq 65$ years seen in ED

\begin{tabular}{|l|}
\hline Total number of discharge Rx for patients $\geq 65$ \\
\hline Number of Beers list Rx prescribed
\end{tabular}

Percentage of Beers list Rx prescribed

\section{Provider 4}

\begin{tabular}{|l|r|r|r|r|r|r|r|r|r|r|r|r|r|r|r|r|}
\hline Number of patients $\geq 65$ years seen in ED & 50 & 37 & 56 & 143 & 48 & 29 & 35 & 112 & 38 & 34 & 34 & 106 & 45 & 40 & 31 & 116 \\
\hline
\end{tabular}

\begin{tabular}{|c|c|c|c|c|c|c|c|c|c|c|c|c|c|c|c|c|}
\hline Total number of discharge Rx for patients $\geq 65$ & 113 & 75 & 133 & 321 & 118 & 62 & 79 & 259 & 74 & 104 & 91 & 269 & 87 & 67 & 66 & 220 \\
\hline Number of Beers list Rx prescribed & 9 & 11 & 6 & 26 & 4 & 6 & 11 & 21 & 5 & 3 & 2 & 10 & 5 & 2 & 2 & \\
\hline
\end{tabular}

Number of Beers list Rx prescribed

Percentage of Beers list Rx prescribed

\begin{tabular}{l|r|r|r|r|r|r|}
8.0 & 14.7 & 4.5 & 8.1 & 3.4 & 9.7 & 13.9 \\
\hline
\end{tabular}

\section{Provider 5}

\begin{tabular}{|l|l|l|l|l|l|l|l|l|l|l|l|l|l|l|l|l|}
\hline Number of patients $\geq 65$ years seen in ED & 60 & 34 & 49 & 143 & 33 & 42 & 43 & 118 & 51 & 64 & 38 & 153 & 53 & 37 & 38 & 128 \\
\hline
\end{tabular}

\begin{tabular}{|l|r|r|r|r|r|r|r|r|r|r|r|r|r|r|r|r|r|r|r|r|r|}
\hline Total number of discharge Rx for patients $\geq 65$ & 99 & 58 & 87 & 244 & 63 & 92 & 61 & 216 & 82 & 77 & 76 & 235 & 94 & 52 & 59 & 205 \\
\hline
\end{tabular}

Number of Beers list Rx prescribed

Percentage of Beers list Rx prescribed

\begin{tabular}{r|r|r|r}
9 & 6 & 7 & 22 \\
9.1 & 10.3 & 8.0 & 9.0
\end{tabular}

\begin{tabular}{r|r|r|r|}
4 & 7 & 4 & 15 \\
\hline 6.3 & 7.6 & 6.6 & 6.9
\end{tabular}

\begin{tabular}{|r|r|r|r|}
\hline 6 & 2 & 4 & 12 \\
\hline 7.3 & 2.6 & 5.3 & 5.1 \\
\hline
\end{tabular}

\begin{tabular}{r|r|r|r|}
7 & 2 & 1 & 10 \\
\hline 7.5 & 3.9 & 1.7 & 4.9 \\
\hline
\end{tabular}

\section{Provider 6}

Number of patients $\geq 65$ years seen in ED

Total number of discharge Rx for patients $\geq 65$

Number of Beers list Rx prescribed

Percentage of Beers list Rx prescribed

ED overall provider

\begin{tabular}{|l|r|r|r|r|r|r|r|r|r|r|r|r|r|r|r|r|}
\hline Number of patients $\geq 65$ years seen in ED & 632 & 519 & 620 & 1,771 & 603 & 545 & 571 & 1,719 & 620 & 620 & 561 & 1,801 & 571 & 565 & 542 & 1,678 \\
\hline
\end{tabular}

\begin{tabular}{|l|l|r|r|r|r|r|r|r|r|r|r|r|r|r|r|r|}
\hline Total number of discharge Rx for patients $\geq 65$ & 1,102 & 902 & 1,103 & 3,107 & 1,036 & 929 & 847 & 2,812 & 1,033 & 890 & 825 & 2,748 & 836 & 840 & 851 & 2,527 \\
\hline Nat
\end{tabular}

Number of Beers list Rx prescribed

Percentage of Beers list Rx prescribed

September 2014

\begin{tabular}{r|r|r|r|r|r|r}
80 & 75 & 77 & 232 & 61 & 82 \\
\hline
\end{tabular}

Provider 1 relative Beers contribution

Provider 2 relative Beers contribution

Provider 3 relative Beers contribution

Provider 4 relative Beers contribution

Provider 5 relative Beers contribution

Provider 6 relative Beers contribution

\begin{tabular}{l|r|r|r|r|}
\hline 7.3 & 8.3 & 7.0 & 7.5 & 5.9 \\
\hline
\end{tabular}

a Select providers prescribing data shown, not all values will add up to $100 \%$

EQUiPPED = Enhancing Quality of Prescribing Practices for Veterans Discharged from the Emergency Department; FY=fiscal year; $Q=q u a r t e r ; R x=m e d i c a t i o n s$. 


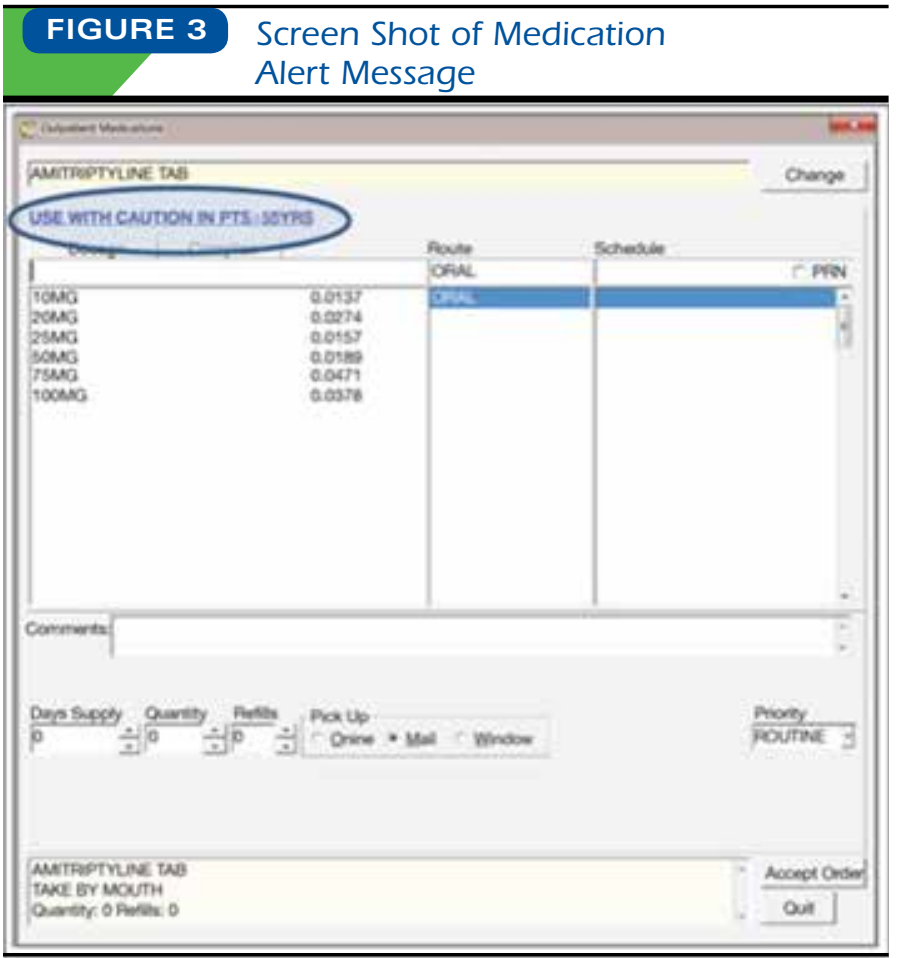

$m g=$ milligram; $P R N=$ as needed; $p t s=$ patients; $t a b=$ tablet; $y r s=$ years .

Automated Provider Prescribing Reports. Beginning in February 2013, providers received monthly reports detailing their discharge prescribing practices in the ED for adults aged 65 years and older. Reports included aggregate data on the individual provider, including patients and prescriptions, number of PIMs, and rate of PIMs on all prescriptions. As provider reports evolved, de-identified ED providers and medical residents as comparators were included for the provider's review. Further information included monthly summaries of all PIMs prescribed, PIMs by age, and the contributions of each provider to the total PIMs prescribed within the month for all prescribers (Figure 2). Additional academic detailing as needed was provided in a correspondence that contained the provider prescribing reports. Data were extracted from VA data warehouses linked to CPRS and computerized physician order entry records for identification of prescribers within the ED clinic stops, evidence of progress note associated with ED discharge, and individual patients aged 65 years and older. A CPS with formulary management expertise led automation efforts to reduce potential errors of omission in identification of incident medication use with the development of a PIMs look-up table. The modified table was then imported into a database and used to identify, collate, and automate the described reports to reduce the delay in time of finalizing provider reports and the associated administrative time.

\section{FIGURE 4 Piecewise Nonlinear Regression Model}

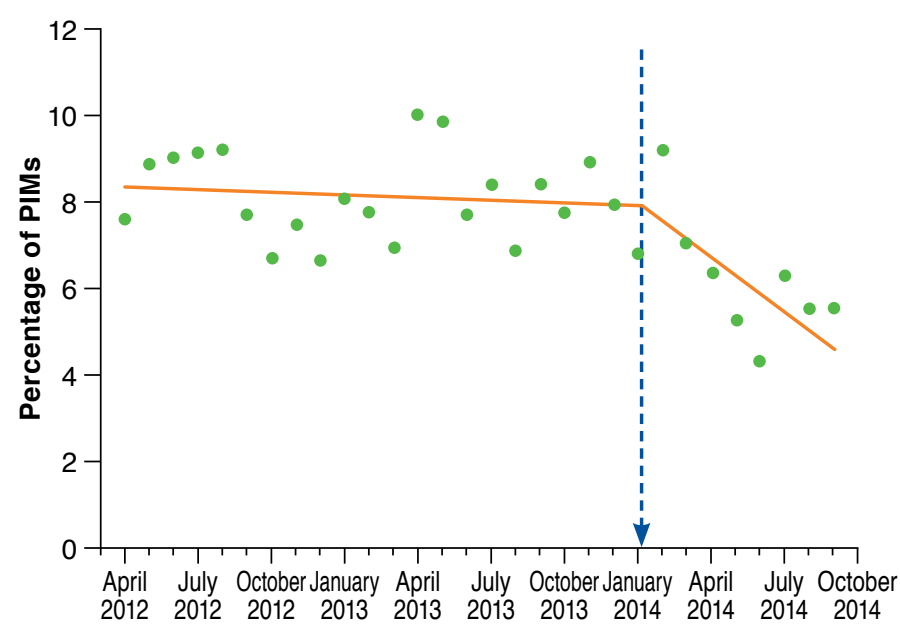

PIMs = potentially inappropriate medications.

Medication Alert Messages. The existing intervention to identify PIMs was dependent upon provider memory from didactic sessions or active use of provided resources. To enhance the impact, the need for an additional reminder was recognized to reinforce the provider's ability to identify medications as potentially inappropriate. The medication alert message (MAM) intervention provided an alternative for interacting with the provider at computerized order entry to identify a medication as potentially inappropriate in patients aged 65 years and older. Eighty-three medications were targeted for the alert as potentially inappropriate. When one of these medications was being ordered, a message appeared as follows: "USE WITH CAUTION IN PTS $\geq 65$ YRS." The length of the message was limited by the number of characters to ensure visual print of message and limit additional provider steps to follow a link. To highlight the alert, all caps, a blue font, and underlined text was used (Figure 3). The MAM was approved by the Pharmacy and Therapeutics Committee and implemented in June 2013.

\section{PIM Rates}

A total of 21,526 medications were prescribed to 23,168 adults aged 65 years and older who were treated and released from the ED between February 2013 and November 2014; 2,684 of these medications were identified as PIMS. The top 5 most frequently prescribed PIMs at baseline were cyclobenzaprine, naproxen, ibuprofen, indomethacin, and methocarbamol. After project implementation, the proportion of prescriptions decreased for all except methocarbamol. Cyclobenzaprine decreased with a $51 \%$ relative reduction, while naproxen, ibuprofen, and indomethacin decreased with 38\%, 52\%, and 7\% relative reductions, respectively. Monthly PIM rates prior to 
project implementation varied from $7.0 \%$ to $9.3 \%$ from January 2012 to January 2013 and from 7.9\% in February 2013 to 4.9\% in November 2014 during the QI initiative. Figure 4 shows the rate of PIMs prescribed monthly from April 2012 through September 2014 with the results of a piecewise, nonlinear regression model. The model indicated that the inflection point occurred in January 2014 after which the rate of PIM prescribing declined more sharply.

\section{Discussion}

This clinical report details the CPS innovations at 1 VA facility in a multisite intervention to decrease PIMs and reports results from 1 participating medical center. Through CPS involvement, this innovative QI intervention was tailored to meet the needs of 1 specific VA ED through the development of 3 key innovations: academic detailing performed by a physician-CPS pair, automated provider reports, and MAM. Our findings reinforce the role of clinical pharmacists and the importance of their contribution to QI projects that center on pharmacy systems in changing prescribing patterns. The facility has experienced a meaningful decrease in the rate of PIM prescribing in older adults discharged from a VA ED. The monthly rate of PIM prescriptions varied but revealed a decreasing trend up to the inflection point, at which point the rate declined more sharply to the lowest rates of PIM prescribing at that point in the project.

The multilayered intervention was implemented at various times during the project. Interestingly, the data indicate January 2014 as the inflection point, after which the rate of PIM prescribing showed a large decrease. The timing of this inflection point can be explained by several important study interventions that also occurred around this time period. First, the inflection point coincided with the period after implementation of the order sets and placement of computer cards identifying the most commonly prescribed PIMs. This was soon followed by the initiation of the automated provider reports. Feedback from ED providers indicated that they found the provider reports to be particularly helpful in evaluating and changing their own individual prescribing practices. In addition, the start of the EM CPS in November 2013 ensured a consistent CPS presence in the ED and had a large impact on avoidance of PIMs prescribing. Also of note, the ED clinic attending physician was the physician in the physician-CPS pair after the inflection point, which, as a supervisor, may have had an impact on changing and sustaining prescribing behaviors.

Results from our QI project show that tailored education and provider feedback, as part of a multilayered intervention, has an impact on the prescribing practices in those health care team members who lack formal geriatrics training. Our report offers an innovative education model that aligns with the Institute of Medicine's (as well as other health disciplines) call for an increase in geriatric education to produce competent health care professionals in the care of the older adult. ${ }^{1,15}$ Given that older adults frequently find themselves in EDs, frequently take multiple chronic medications, ${ }^{16,17}$ and have pharmacokinetic and pharmacodynamic age-related changes that increase risk of adverse drug events, ${ }^{18}$ specialized education such as that provided for in our model is important to ensure safe prescribing to older adults in an ED environment.

Future directions include expanding this QI project to other VA EDs. During the QI project, a need was identified for an innovative technological tool to export the key innovations to other VA EDs that do not possess the infrastructure or personnel at their local institutions. The concept of dashboards as a potential means for dissemination was introduced by CPS during the initiative. Dashboards are innovative data packages that generate a number of key indicators with the ability to be modified to tailor individual data needs. Through automation of provider feedback reports on prescribing practices and dissemination of EQUiPPED products, implementing this project would be easily portable to other VA EDs. Electronic accessibility through VA Internet share points or other database tools would allow for ED providers to view their own data and prescribing habits. The dashboard would have the capability to allow for interactive data retrieval tailored specifically to providers to not only provide information on peer comparisons, but also to be a passive educational tool that could be reviewed by prescribers or an active tool used for detailing similar to the physician-CPS pairing that has been described in this article. The feasibility of this concept is currently being explored and tested.

\section{Limitations}

Some limitations of this report should be noted. Given the pre/post design, we cannot exclude the possibility that interventions other than EQUiPPED contributed to declining PIM rates. Additionally, we are unable to determine the impact of individual components of the overall intervention, although the piecewise logistic regression gives us some clues. Also, although our data show a decrease in Beer's prescribing, we are unable to say whether or not this had an impact on other important clinical outcomes such as adverse drug events, re-presentations to the ED, or hospitalizations. These are all important areas for future inquiry.

\section{Conclusions}

The QI project resulted in a meaningful decrease in PIM prescribing in older ED adults. CPS contributions to QI can extend beyond pharmacotherapy and provider education to also include information technology tools using formulary management expertise. 


\section{Authors}

JASON M. MOSS, PharmD, CGP, is Assistant Professor of Pharmacy Practice, Campbell University College of Pharmacy and Health Sciences, and Clinical Pharmacy Specialist, Geriatric Research Education and Clinical Center, Veterans Affairs Medical Center, Durham, North Carolina. WILLIAM E. BRYAN, III, PharmD, BCPS, is Clinical Pharmacy Specialist, and RYAN K. OWENBY, PharmD, is Clinical Pharmacy Specialist, Pharmacy Service, Veterans Affairs Medical Center, Durham, North Carolina. LOREN M. WILKERSON, MD, is Medical Instructor, Duke University School of Medicine, Durham, North Carolina. GEORGE L. JACKSON, PhD, MHA, is Research Health Scientist, Center for Health Services Research in Primary Care, Veterans Affairs Medical Center, and Associate Professor, Duke University School of Medicine, Durham, North Carolina. COURTNEY H. VAN HOUTVEN, PhD, is Associate Professor, Duke University School of Medicine, and Research Scientist, Center for Health Services Research in Primary Care, Veterans Affairs Medical Center, Durham, North Carolina. MELISSA B. STEVENS, MD, is Assistant Professor, Emory University School of Medicine, and Affiliate Investigator, Birmingham/Atlanta Geriatric Research Education and Clinical Center, Atlanta VA Medical Center, Atlanta, Georgia. CAMILLE P. VAUGHAN, MD, MS, is Assistant Professor, Emory University School of Medicine, and Investigator, Birmingham/ Atlanta Geriatric Research Education and Clinical Center, Atlanta VA Medical Center, Atlanta, Georgia. JAMES S. POWERS, MD, is Associate Clinical Director, Geriatric Research Education and Clinical Center, Tennessee Valley Healthcare System, and Associate Professor, Vanderbilt University School of Medicine, Nashville, Tennessee. WILLIAM W. HUNG, MD, MPH, is Assistant Professor, Icahn School of Medicine at Mount Sinai, and Associate Directorl Clinical (Acting), Geriatric Research Education and Clinical Center, Veterans Affairs Medical Center, Bronx, New York. ULA HWANG, MD, MPH, is Assistant Professor, Icahn School of Medicine at Mount Sinai, and Associate Director/Clinical (Acting), Geriatric Research Education and Clinical Center, Veterans Affairs Medical Center, Bronx, New York. ALAYNE D. MARKLAND, DO, MSc, is Investigator, Department of Veterans Affairs Birmingham, Birmingham, Alabama/Atlanta Geriatric Research, Education, and Clinical Center, and Assistant Professor, University of Alabama School of Medicine at Birmingham. GERALD MCGWIN, MS, $\mathrm{PhD}$, is Investigator, Department of Veterans Affairs Birmingham, Birmingham, Alabama/Atlanta Geriatric Research, Education, and Clinical Center; Professor and Vice Chairman, Department of Epidemiology; and Director, Biostatistics, Epidemiology and Research Design, Center for Clinical and Translational Science, University of Alabama at Birmingham. SUSAN NICOLE HASTINGS, MD, MHS, is Investigator, Geriatric Research Education and Clinical Center, Veterans Affairs Medical Center, Durham, North Carolina; Associate Professor, Duke University School of Medicine; and Investigator, Center for Health Services Research in Primary Care, Veterans Affairs Medical Center, Durham, North Carolina.

AUTHOR CORRESPONDENCE: Jason M. Moss, PharmD, CGP, Geriatric Research Education and Clinical Center, Durham VA Medical Center, 508 Fulton St., GRECC/182, Durham, NC 27705. Tel.: 919.286.0411, ext. 7878; Fax: 919.286.6823;

E-mail: jason.moss@va.gov.

\section{DISCLOSURES}

Funding was provided by the Department of Veterans Affairs Office of Geriatrics and Extended Care T-21 initiative (G508-1 \& G521-5). Vaughan is supported by a Rehabilitation R\&D CDA-2 award from the Department of Veterans Affairs 1 IK2 RX000747-01. The views expressed in this article are those of the authors and do not necessarily represent the views of the Department of Veterans Affairs. The authors report no conflicts of interest. Portions of this work were presented at the Annual Scientific Meeting of the American Geriatrics Society meeting in Orlando, Florida, on May 16, 2014.

Study design was created by Stevens, Powers, Vaughan, and Hastings, with assistance from Moss. Bryan, Wilkerson, Jackson, and Owenby collected the data, and data interpretation was performed by Van Houtven, McGwin, and Hastings, with assistance from Moss, Owenby, and Stevens. The manuscript was written by Moss and Hastings and revised by Moss, Hung, Hwang, and Markland.

\section{ACKNOWLEDGMENTS}

The authors would like to acknowledge Sherman Lee for providing editorial support.

\section{REFERENCES}

1. Delafuente JC. The silver tsunami is coming: will pharmacy be swept away with the tide? Am J Pharm Educ. 2009;73(1):1.

2. Hastings SN, Schmader KE, Sloane RJ, et al. Quality of pharmacotherapy and outcomes for older veterans discharged from the emergency department. J Am Geriatr Soc. 2008;56(5):875-80.

3. Hafner JW Jr, Belknap SM, Squillante MD, Bucheit KA. Adverse drug events in emergency department patients. Ann Emerg Med. 2002;39(3):258-67.

4. The American Geriatrics Society 2012 Beers Criteria Update Expert Panel. American Geriatrics Society updated Beers Criteria for potentially inappropriate medication use in older adults. J Am Geriatr Soc. 2012;60(4):616-31.

5. Caterino JM, Emond JA, Camargo CA Jr. Inappropriate medication administration to the acutely ill elderly: a nationwide emergency department study, 19922000. J Am Geriatr Soc. 2004;52(11):1847-55.

6. Koyama A, Steinman M, Ensrud K, Hillier TA, Yaffe K. Long-term cognitive and functional effects of potentially inappropriate medications in older women. $J$ Gerontol A Biol Sci Med Sci. 2014;69(4):423-29.

7. Thorpe JM, Thorpe CT, Kennelty KA, Gellad WF, Schulz R. The impact of family caregivers on potentially inappropriate medication use in noninstitutionalized older adults with dementia. Am J Geriatr Pharmacother. 2012;10(4):230-41. 8. Aldridge VE, Park HK, Bounthavong M, Morreale AP. Implementing a comprehensive, 24-hour emergency department pharmacy program. Am J Health Syst Pharm. 2009;66(21):1943-47.

9. Aspinall SL, Cunningham FE, Zhao X, et al. Impact of pharmacist-managed erythropoiesis-stimulating agents clinics for patients with non-dialysis-dependent CKD. Am J Kidney Dis. 2012;60(3):371-79.

10. Marino EL, Alvarez-Rubio L, Miro S, et al. Pharmacist intervention in treatment of patients with genotype 1 chronic hepatitis C. J Manag Care Pharm. 2009;15(2):14750. Available at: http://www.amcp.org/data/jmcp/147-150.pdf.

11. Smith JP, Dong MH, Kaunitz JD. Evaluation of a pharmacist-managed hepatitis C care clinic. Am J Health Syst Pharm. 2007;64(6):632-36.

12. Geber J, Parra D, Beckey NP, Korman L. Optimizing drug therapy in patients with cardiovascular disease: the impact of pharmacist-managed pharmacotherapy clinics in a primary care setting. Pharmacotherapy. 2002;22(6):738-47.

13. Stevens M, Hastings SN, Powers J, et al. Enhancing the Quality of Prescribing Practices for Older Veterans Discharged from the Emergency Department: preliminary results from Enhancing Quality of Prescribing Practices for Older Veterans Discharged from the Emergency Department,

a novel multi-component interdisciplinary quality improvement initiative. J Am Geriatr Soc. 2015;63(5):1025-29.

14. Kessler C, Chen J, Dill C, Tyndall G, Olszyk MD. State of affairs of emergency medicine in the Veterans Health Administration. Am J Emerg Med. 2010;28(8):947-51

15. Institute of Medicine. Retooling for an Aging America: Building the Health Care Workforce. Washington, DC: National Academies Press; 2008.

16. Qato DM, Alexander GC, Conti RM, Johnson M, Schumm P, Lindau ST. Use of prescription and over-the-counter medications and dietary supplements among older adults in the United States. JAMA. 2008;300(24):2867-78.

17. Hajjar ER, Hanlon JT, Sloane RJ, et al. Unnecessary drug use in frail older people at hospital discharge. J Am Geriatric Soc. 2005;53(9):1518-23.

18. Hanlon JT, Sloane RJ, Pieper CF, Schmader KF. Association of adverse drug reactions with drug-drug and drug-disease interactions in frail older outpatients. Age Ageing. 2011;40(2):274-77. 